\title{
Diffusion-Weighted MR Imaging and MGMT Methylation Status in Glioblastoma: A Reappraisal of the Role of Preoperative Quantitative ADC Measurements
}

M ethylation of the DNA repair enzyme O6-methylguanineDNA-methyltransferase (MGMT) has been well described as one the most significant biomarkers of glioblastoma (GBM) patient prognosis and response to standard first-line chemotherapy treatment with temozolomide. ${ }^{1}$ As such, we read with great interest the recent study published in AJNR in May 2011 entitled "Apparent Diffusion Coefficient Histogram Analysis Stratifies Progression-Free Survival in Newly Diagnosed BevacizumabTreated Glioblastoma" by Dr. Pope and colleagues. ${ }^{2}$

A significant conclusion of this study was that "lower ADC is associated with tumor MGMT promoter methylation." This is a finding of significant interest to radiologists and oncologists alike as it suggests that ADC measures can potentially function as both a prognostic and predictive imaging biomarker and thereby act as a surrogate for the reference standard pathologic determination of MGMT methylation status. The authors arrived at this conclusion based on a pixel-by-pixel ADC histogram analysis with bimodal curve fitting of enhancing tumor in 89 patients with GBM with pathologically confirmed methylation status. This analysis showed a mean ADC of $1071 \times 10^{-6} \mathrm{~mm}^{2} / \mathrm{s}$ for 36 methylated tumors versus $1183 \times 10^{-6} \mathrm{~mm}^{2} / \mathrm{s}$ for 53 unmethylated tumors, with a $P$ value of .01 between the groups.

To assess the applicability of these findings to our own patients, we retrospectively performed blinded quantitative ADC measurements in 105 treatment naïve, preoperative patients with GBM with pathologically confirmed MGMT promoter methylation status determined through real-time methylation specific polymerase chain reaction. Our goal was to build on the work of Pope et $\mathrm{al}^{2}$ by using an ADC quantification technique readily

http://dx.doi.org/10.3174/ajnr.A3467

Table 1: Relationship between ADC metrics and MGMT status

\begin{tabular}{|c|c|c|c|}
\hline \multirow[b]{2}{*}{ Diffusion MRI } & \multicolumn{2}{|c|}{ MGMT Methylation Status (median, range) } & \multirow[b]{2}{*}{$P$ Value } \\
\hline & No $(n=66)$ & Yes $(n=39)$ & \\
\hline $\mathrm{ADC}_{\text {mean }}$ & $0.00120(0.00012-0.00177)$ & $0.00123(0.00011-0.00197)$ & .48 \\
\hline$A D C_{\min }$ & $0.00085(0.00016-0.00838)$ & $0.00084(0.00054-0.00117)$ & .85 \\
\hline$A D C_{\max }$ & $0.00184(0.00102-0.00289)$ & $0.00212(0.00086-0.00328)$ & .12 \\
\hline$A D C_{R O I}$ & $0.00097(0.00067-0.00159)$ & $0.00099(0.00068-0.00147)$ & .91 \\
\hline$A D C_{\text {ratio }}$ & $1.30144(0.90670-1.90337)$ & $1.23086(0.18519-2.33216)$ & .29 \\
\hline
\end{tabular}

ADC expressed as $\mathrm{mm}^{2} / \mathrm{s}$ 


\section{REFERENCES}

1. Kim YS, Kim SH, Cho J, et al. MGMT gene promoter methylation as a potent prognostic factor in glioblastoma treated with temozolomide-based chemoradiotherapy: a single-institution study. Int $J$ Radiat Oncol Biol Phys 2012;84:661-67

2. Pope WB, Lai A, Mehta R, et al. Apparent diffusion coefficient histogram analysis stratifies progression-free survival in newly diagnosed bevacizumab-treated glioblastoma. AJNR Am J Neuroradiol 2011;32:882-89

3. Law M, Young R, Babb J, et al. Histogram analysis versus region of interest analysis of dynamic susceptibility contrast perfusion MR imaging data in the grading of cerebral gliomas. AJNR Am J Neuroradiol 2007;28:761-66

4. Wetzel SG, Cha S, Johnson G, et al. Relative cerebral blood volume measurements in intracranial mass lesions: interobserver and intraobserver reproducibility study. Radiology 2002;224:797-803

A. Gupta

Department of Radiology

Weill Cornell Medical College/New-York Presbyterian Hospital

New York, New York

A. Prager

R.J. Young

Department of Radiology

w. Shi

Department of Epidemiology and Biostatistics

A.M.P. Omuro

Department of Neurology

Memorial Sloan-Kettering Cancer Center

New York, New York

J.J. Graber

Department of Neurology

Montefiore Medical Center

New York, New York 